\title{
Audit of surgical procedures in regional and central hospitals in Limpopo Province, South Africa
} \author{
A B van As, ${ }^{3,4} \mathrm{MB}$ ChB, MMed, MBA, FCS (SA), PhD \\ ${ }^{1}$ Department of Health, Polokwane, South Africa \\ ${ }^{2}$ Department of Information Management, Pietersburg Hospital, Polokwane, South Africa \\ ${ }^{3}$ Department of General Surgery, Pietersburg Hospital, Polokwane, South Africa \\ ${ }^{4}$ Department of Surgery, University of Limpopo, Polokwane, South Africa
}

S Ngwenya, ${ }^{1}$ MSc; R Mavhungu, ${ }^{2}$ BSc; M K Mokwena, ${ }^{1}$ BCur, PG Dip Public Health; A Robertson, ${ }^{1}$ MB ChB, FC Paeds;

Corresponding author: A B van As (sebastian.vanas@uct.ac.za)

Background. There is a paucity of data on the functioning and surgical procedures performed in rural hospitals in South Africa. Objective. To determine the category of procedures performed at regional and tertiary hospitals in Limpopo Province, South Africa. Method. We conducted a retrospective analysis of surgical procedures performed in the regional and tertiary hospitals in Limpopo Province during a 1-year period from 1 March 2019 to 29 February 2020.

Results. A total of 24263 surgical procedures were performed during the study period. More than half of all cases $(50.4 \% ; n=12252)$ were operated on at the tertiary hospitals while regional hospitals performed the remaining 12011 operations.

Conclusion. There is a great necessity to alleviate the central hospitals from the bulk of surgical procedures.

S Afr Med J 2021;111(11b):1126-1128.https://doi.org/10.7196/SAMJ.2021.v111.i11b.16123

Limpopo Province is one of the most surgeon-deprived provinces in South Africa (SA). This represents a major challenge for healthcare, particularly for patients who require surgical procedures. Besides the serious shortage of surgical specialists, the vast geographic outlay of the province makes it very difficult for patients to reach a hospital where surgical procedures are offered. This results in delays in seeking medical attention, and in patients often arriving at very late stages of disease, further complicating effective surgical management. Surgical care is considered to be an integral part of health systems worldwide and these delays are tragic. ${ }^{[1]}$

The Limpopo Province covers an area of $125754 \mathrm{~km}^{2}$ and is populated by 5.98 million people. ${ }^{[2]}$ The province is subdivided into five districts: Capricorn, Vhembe, Mopani, Sekhukhune and Waterberg. The majority of people in the province reside in rural areas. Each district has one regional hospital and a number of district hospitals. The province has two tertiary hospitals which are $30 \mathrm{~km}$ apart. The tertiary hospitals are in Capricorn district. One of the two tertiary hospitals is at Polokwane, the provincial capital.

Auditing surgical services provision is important, especially in rural provinces like Limpopo, where the minimal available resources are targeted at operational services, depriving local research activities. Auditing allows for improvement in the quality of services offered and identification of service areas that need improvement. ${ }^{[3]}$ A study conducted in the Pietermaritzburg tertiary hospital found that $30 \%$ of their workload comprised operations that could be performed at a district level. ${ }^{[4]}$ This finding concurs with the observation made by Bhuiyan and Mavhungu, ${ }^{[5]}$ namely that hernia repairs and amputations were found to be the most common procedures performed on a Saturday list at Pietersburg Hospital (PH).

The provincial Department of Health has been trying to be proactive in launching a number of programmes aimed at alleviating the shortage of surgeons in the province. There is the surgical outreach programme where surgical specialists visit regional or district hospitals in a group to assist local surgeons to reduce the waiting list for patients who require surgery. Specialists involved include reproductive medicine specialists, urogynaecological specialists, plastic and reconstructive surgeons, paediatric surgeons, ENT surgeons, general surgeons, radiologists, maxillofacial surgeons, orthopaedic surgeons, urologists, ophthalmologists, cardiologists and anaesthetists.

The other programme (National Health Insurance grant AOS001/19/20) consists of accreditation of service providers for the provisioning of specialised clinical and surgical services for the Limpopo Department of Health for a period of 36 months. A specific grant was allocated for the 2019 - 2022 period to address surgical backlog in the province utilising private healthcare institutions.

\section{Methods}

We conducted a retrospective descriptive record review of patients admitted to the regional and tertiary hospitals for operations in the period 1 March 2019 - 29 February 2020. The two academic hospitals are Pietersburg and Mankweng hospitals ( $\mathrm{PH}$ and $\mathrm{MH}$ ), and the five regional hospitals are Letaba, Mokopane, Philadelphia, St Rita's and Tshilidzini. All patients admitted to these hospitals for operations during the audit period were included in the present study. The operations included general surgery, nephrology, orthopaedics, urology, neurosurgery, ear, nose and throat (ENT), cardiothoracic, plastic and reconstructive, ophthalmology, maxillofacial, gynaecology, obstetrics and paediatric surgery. Theatre registers were used as a starting point for data collection. A data collection sheet designed for the audit was used to collect information from the theatre registers. A password-protected database in Excel (Microsoft Corp., USA) was created to record the information regarding operated patients. The integrity of the data was verified through an evaluation of internal consistency and assessment of missing data.

The statistical package in Excel (Microsoft Corp., USA) was used for data analysis. The categorical variables were described as proportions. 


\section{Results}

\section{General results}

A total of 24263 surgical procedures were entered into the database during 2019 - 2020. The surgical patients admitted and operated on for the 1-year period are shown in Fig. 1. More than half of the patients $(50.4 \% ; n=12252)$ were admitted and operated on at the tertiary hospitals while regional hospitals performed 12011 operations. Ophthalmology comprised over $41 \%(n=2301)$ of the surgical workload at MH (Table 1).

\section{Obstetrics and gynaecology}

Obstetrics and gynaecology comprised the majority (44.5\%; $n=10768$ ) of the total operations. Regional hospitals performed $72 \%$ of the obstetrics and gynaecology procedures (Fig. 2).

\section{General surgery}

We found that 4335 patients were operated in the various departments of general surgery. Tertiary hospitals contributed half $(50 \% ; n=2092)$ of the procedures performed in general surgery (Fig. 3). The majority (50.1\%) of general surgery patients admitted and operated at the regional hospitals were from Letaba Hospital. Tshilidzini Hospital performed fewer operations than Letaba Hospital during 2019 - 2020. Mokopane (3.8\%) and Philadelphia (0.8\%) contributed a relatively small number of operations ( $n=197$; Fig. 3 ).

Table 1. Surgical procedures performed at tertiary hospitals

\begin{tabular}{lll}
\hline & $\begin{array}{l}\text { Mankweng } \\
\text { Hospital, } \boldsymbol{n}\end{array}$ & $\begin{array}{l}\text { Pietersburg } \\
\text { Hospital, } \boldsymbol{n}\end{array}$ \\
\hline General surgery & 973 & 1155 \\
Gynaecology & 460 & 816 \\
Orthopaedic & 1031 & 1021 \\
Urology & 2 & 420 \\
ENT & 0 & 498 \\
Neurosurgery & 42 & 583 \\
Plastic surgery & 187 & 157 \\
Maxillofacial & 68 & 150 \\
Obstetrics & 231 & 1494 \\
Cardiothoracic & 62 & 230 \\
Nephrology & 0 & 130 \\
Paediatric surgery & 291 & 19 \\
Ophthalmology & 2301 & 0 \\
Total & 5576 & 6676 \\
ENT = ear, nose and throat. & &
\end{tabular}

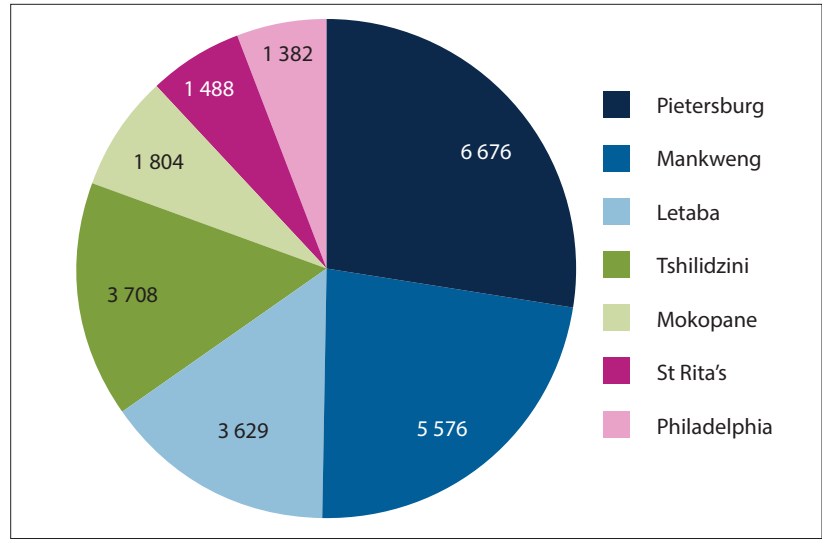

Fig. 1. Total number of surgical procedures performed in Limpopo regional and academic hospitals from 1 March 2019 to 29 February 2020.

\section{Orthopaedic surgery}

We found that 4056 of patients who were operated required orthopaedic attention. Tertiary $(n=2055)$ and regional $(n=2001)$ hospitals contributed nearly the same number of procedures during the audit period. Mokopane and Tshilidzini hospitals contributed more than half $(52 \%)$ of the operations performed in regional hospitals, and Philadephia Hospital contributed only 13.7\% (Fig. 4).

\section{Analysis of surgical procedures performed at the tertiary hospitals}

Although the two tertiary hospitals have almost the same number of special units, nearly the same number of beds and theatres, the majority of operations were performed at PH (54\%; $n=6$ 676). Ophthalmology contributed more than $41 \%$ of operations performed at $\mathrm{MH}$ during the audit period. $\mathrm{MH}$ is one of only two facilities in the province rendering ophthalmology services.

\section{Discussion}

This audit presents the distribution of surgical procedures performed in the rural province of Limpopo during 2019 - 2020. Over half $(50.4 \%)$ of the operations were of patients admitted and managed at tertiary hospitals (Fig. 1). This is way above figures recorded in other studies conducted in SA. A study by van Straten ${ }^{[6]}$ reported that $29 \%$ of patients discharged from Charlotte Maxeke Johannesburg Academic Hospital Department of Surgery were suitable for secondary level

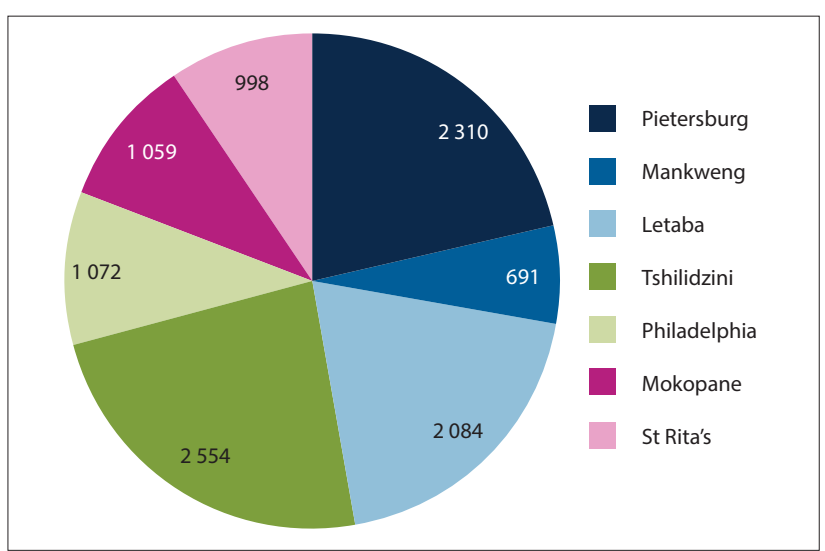

Fig. 2. Total number of surgical procedures performed in the general surgical departments in regional and academic hospitals in Limpopo Province from 1 March 2019 until 29 February 2020.

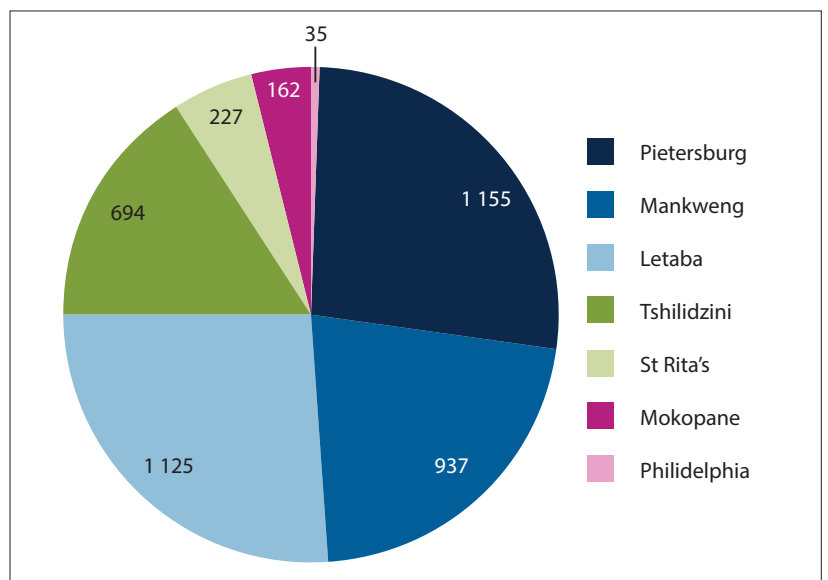

Fig. 3. Total number of surgical procedures performed in the obstetrics and gynaecology departments of regional and academic hospitals in Limpopo Province from 1 March 2019 until 29 February 2020. 


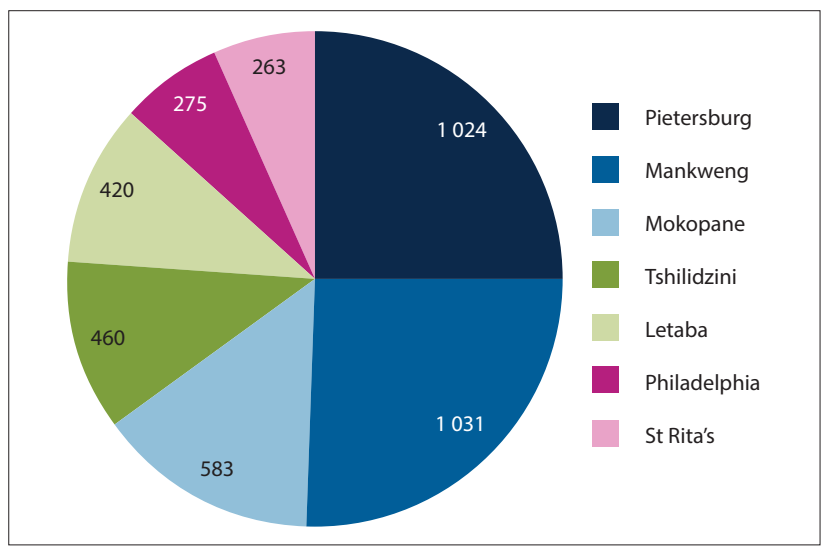

Fig. 4. Total number of surgical procedures performed in the orthopaedic surgical departments in regional and academic hospitals in Limpopo Province from 1 March 2019 until 29 February 2020.

care ${ }^{[6]}$ Lack of skills and resources in the public healthcare system has a tendency to result in upward referral due to local constraints. ${ }^{[4]}$ This is particularly the case in surgery, which requires intricate healthcare systems to function more adequately. All operations are highly complex procedures, where the infrastructure (physical theatres) and the multitude of personnel (cleaners, nurses, anaesthetic staff and surgeons) all have to be fine-tuned to perfection to accommodate the patient. ${ }^{[1]}$ Inappropriate upward referral of surgical patients is definitely not cost effective, since tertiary beds are approximately twice as expensive to run compared with beds in regional hospitals. ${ }^{[5]}$

More than $40 \%$ of surgical procedures performed at the tertiary and regional hospitals during the study period were obstetrics and gynaecology. The proportion of obstetrics and gynaecology operations was consistently higher for all regional hospitals, and a total of $72 \%$ of all obstetrics and gynaecology procedures were performed in regional hospitals. More than one-third of obstetrics and gynaecology procedures were performed at Tshilidzini Hospital. St Rita's Hospital contributed a very small number of gynaecology operations.

Although $\mathrm{PH}$ and $\mathrm{MH}$ are both tertiary hospitals of the same size (500 beds, 8 theatres each), PH performed more surgical procedures than $\mathrm{MH}$. The majority of the operations performed at $\mathrm{MH}$ were for ophthalmology patients. $\mathrm{PH}$ and $\mathrm{MH}$ share nearly the same orthopaedic workload.

One of the major findings of this study was that $50 \%$ of the general surgery procedures were performed at tertiary hospitals. It was further noted that one regional hospital (Letaba) performed more than half of the total number of operations in regional hospitals, indicating the great disparity in number of operations performed in regional hospital. To empower regional hospitals to perform more cost-effective operations, an in-depth audit is required to identify constraints encountered by some regional hospitals. Philadelphia and St Rita's hospitals consistently contributed the lowest number of procedures for all surgical disciplines.

One of the greatest challenges in dealing with the large number of surgical patients in the central hospitals has been the absence of a dedicated surgical emergency theatre. This has resulted in the surgical departments having to share the emergency theatre with the departments of obstetrics and gynaecology. In addition, if emergency cases fail to be operated during the night and after hours, the unfortunate consequence has been that elective surgical theatre slates are crushed, resulting in very frustrated surgical patients and surgeons as well as increased inefficient hospital bed utilisation. The costs escalate, frustrating the patients, doctors and nurses.

\section{Conclusion}

The present study describes the distribution of surgical workload in Limpopo Province, SA. It is clear that the tertiary institutions perform most of the operations, the majority of which should ideally be performed in regional hospitals. The most common surgical procedures performed are obstetrical and gynaecological. One tertiary hospital $(\mathrm{MH})$ is overloaded with ophthalmology procedures. Although one hospital performed a significant number of general surgery operations (Letaba Hospital), this study indicates that there is a great necessity to alleviate the central hospitals from the bulk of surgical procedures. Key to the solution will be to staff the regional hospitals with adequately trained personnel as well as provide the relevant infrastructure and medical equipment to perform these surgical operations.

\section{Declaration. None.}

Acknowledgements. None.

Author contributions. Equal contributions.

Funding. None.

Conflicts of interest. None.

1. Meara JG, Leather AJM, Hagander L, et al. Global surgery 2030: Evidence and solutions for achievin health, welfare, and economic development. Lancet 2015;386(9993):569-624. https://doi.org/10.1016/ S0140-6736

2. Statistic South Africa. Mid-year population estimates, statistical release P0302. Stats: Pretoria, 2019. www.statssa.gov.za

3. Limb C, Fowler A, Gundogan B, Koshy K, Agha R. How to conduct a clinical audit and quality improvement project. Int J Surg Oncol (NY) 2017;2(6):e24. https://doi.org/10.1097\%2F IJ9.0000000000000024

4. Laing GL, Skinner DL, Bruce JL, et al. Auditing surgical service provision at a South African tertiary institution: Implications for the development of district services. S Afr J Surg 2017;55(4):31-35.

5. Bhuiyan MMZU, Mavhungu R. Provision of an emergency theatre in tertiary hospitals is cost-effective: Audit and cost of cancelled planned elective general surgical operations at Pietersburg Hospital, Limpopo Province, South Africa. SAMJ 2017;107(3):239-242. https://doi.org/10.7196/SAMJ.2017. v107i3.10687.

6. Van Straten S, Stannard C, Bulabula J, Paul K, Leong J, Klipin MJ. A third of patients treated at a tertiar level surgical service could be treated at a secondary level facility. SAMJ 2017;107(9):788-790. https:/ doi.org/10.7196/SAMJ.2017.v107i9.12090

Accepted 8 September 2021 